\title{
Tratamiento de la incontinencia urinaria de esfuerzo post-prostatectomía radical mediante malla anclada a ramas isquiopubianas
}

\author{
J.A. Queipo Zaragozá, F. Chicote Pérez, A. Borrell Palanca, J.F. Beltrán Meseguer, \\ L. García Reboll, F. Pastor Sempere
}

Servicio de Urología, Hospital de Sagunto. Sagunto (Valencia).

Actas Urol Esp 2005; 29 (8): 764-768

\section{RESUMEN}

TRATAMIENTO DE LA INCONTINENCIA URINARIA DE ESFUERZO POST-PROSTATECTOMÍA RADICAL MEDIANTE MALLA ANCLADA A RAMAS ISQUIOPUBIANAS

Presentamos los resultados de una alternativa en el tratamiento de la incontinencia urinaria de esfuerzo masculina post-prostatectomía radical. Se trata de una técnica de renovado interés en los últimos años, basada en el procedimiento de Stamey, consistente en colocar una malla de polipropileno en posición suburetral, anclada mediante tornillos a las ramas isquiopubianas. Hemos aplicado el procedimiento en 4 pacientes oncológicamente estables y con incontinencia urinaria de larga evolución. Tras un seguimiento medio de 12,5 meses, dos pacientes presentan continencia total, uno ha mejorado significativamente y en el otro persiste la incontinencia. No ha habido complicaciones postoperatorias ni rechazo de la malla. A partir de estos resultados preliminares y la bibliografía revisada, pensamos que esta técnica puede constituir una alternativa al esfinter urinario artificial, dados sus resultados clínicos, su facilidad de realización, la buena tolerancia y su bajo coste.

Palabras Clave: Sling suburetral. Incontinencia. Prostatectomia radical.

\section{ABSTRACT}

TREATMENT OF THE STRESS URINARY INCONTINENCE OF MALE RADICAL POSTPROSTATECTOMY ANCHORED BY MEANS OF SCREWS TO THE ISQUIOPUBIAN BRANCHES

We presented the results of an alternative in the treatment of the stress urinary incontinence of male radical postprostatectomy. Is a technique of renewed interest in the last years, cradle in the procedure of Stamey, consisting of placing an on guard suburetral polypropylene mesh, anchored by means of screws to the isquiopubian branches. We have applied the procedure in 4 patients and with stress urinary incontinence of long evolution. After an average pursuit of 12.5 months, two patients present total continence, one has improved significantly and in the other the incontinence persists. There have been no postoperating complications nor rejection of the mesh. From these preliminary results and the reviewed bibliography, we thought that this technique can constitute an alternative to the artificial urinary sphincter, dices its clinical results, its facility of execution, the good tolerance and his low cost.

Keywords: Urethral Sling, Incontinence, Radical Prostatectomy.

$\mathrm{L}$ a practica cada vez mas frecuente de la prostatectomía radical en el tratamiento del cáncer de próstata ha determinado que cada vez sea mas frecuente la aparición de una incontinencia urinaria de esfuerzo (IUE) en el varón. La aparición de la IUE, generalmente debido a una deficiencia esfinteriana intrínseca ${ }^{1}$, es una complicación que puede darse y de lo cual se debe advertir al paciente. Las opciones de tratamiento son diversas, desde la realización de ejercicios de rehabilitación 
del suelo pélvico, pasando por tratamientos farmacológicos, inyección transuretral de sustancias ocupantes de espacio y el esfínter urinario artificial (EUA), siendo éste último el que parece presentar los mejores resultados. Sin embargo, el EUA no está exento de complicaciones, fallos y necesidad de revisiones quirúrgicas conforme pasa el tiempo ${ }^{2}$. Por ello ha tomado un renovado interés la colocación de "slings suburetrales", práctica hasta ahora no muy extendida.

La colocación de una malla en la porción cavernosa de la uretra bulbar requiere un sistema de fijación. Éste puede llevarse a cabo mediante sutura y paso de agujas retropúbicas (como en el procedimiento de Raz en mujeres con IEU) o bien mediante anclaje con tornillos a las ramas isquiopubianas (Fig. 1). En nuestro servicio hemos optado por la segunda técnica, salvando así la posible yatrogenia de las agujas en su trayecto retropúbico. Además, consideramos que el anclaje de la malla al hueso permite una fijación más sólida y resistente. Comenzamos a aplicar esta técnica a partir de los trabajos publicados por Madjar y cols ${ }^{3}$ y Comiter ${ }^{4}$. Presentamos los resultados preliminares de una corta serie de pacientes con IUE post-prostatectomía radical sometidos a este procedimiento.

\section{MATERIAL Y MÉTODOS}

Entre octubre del 2003 y septiembre del 2004 4 pacientes han sido intervenidos mediante colocación de una malla suburetral con anclaje óseo.

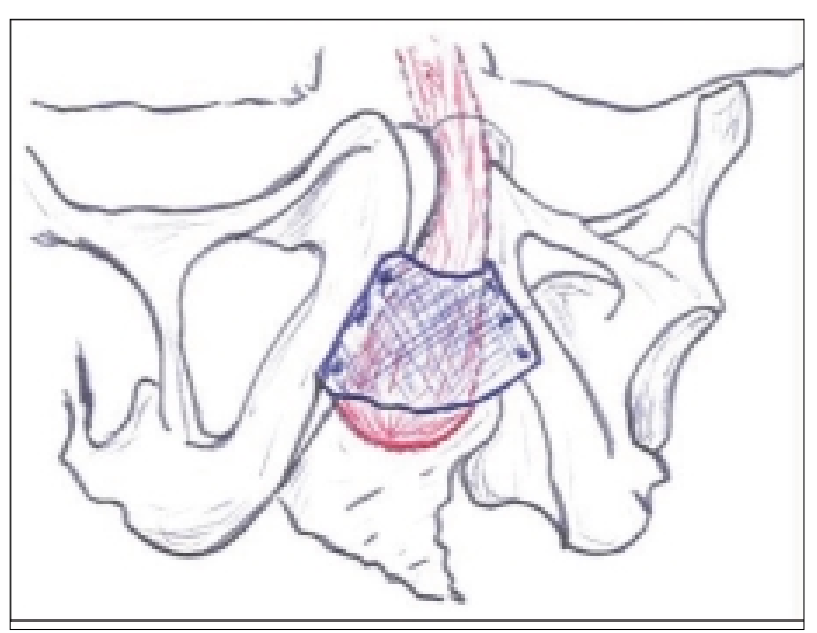

FIGURA 1. Esquema de un sling suburetral con anclaje a ramas isquiopubianas.
Todos ellos presentaban una incontinencia urinaria moderada con importante alteración de su calidad de vida, secundaria a una prostatectomía radical previa. En los dos primeros casos se utilizó una malla de polipropileno de 6 x $10 \mathrm{~cm}$. y 6 tornillos de $5 \mathrm{~mm}$ de diámetro con aparataje proporcionado por el Servicio de Traumatología de nuestro Hospital. En los dos últimos casos utilizamos el kit INVANCE $^{\circledR}$ de American Medical Systems ${ }^{\circledR}$. Todos los pacientes presentaban un largo tiempo libre de enfermedad, sin requerir bloqueo androgénico. Los controles clínicos y de continencia se evaluaron al mes, 3, 6, 12 y 18 meses.

El procedimiento se lleva a cabo con el paciente en posición de litotomía forzada y elevación de la bolsa escrotal. La palpación transperineal ayuda a identificar bilateralmente las tuberosidades isquiáticas, realizando una incisión en rafe perineal medio, desde el escroto hasta la altura de dichas tuberosidades (Fig. 2). Tras disección del tejido subcutáneo y fascia en sentido lateral, identificamos las ramas isquiopubianas hacia el pubis y descubrimos el periostio, sobre el cual se fijaran los tornillos que sujetaran la malla. La disección en la línea media debe restringirse al máximo para evitar la lesión del músculo bulbocavernoso. Comenzando por el lado derecho se insertan en la rama isquiopubiana 3 tornillos de titanio de 3 ó $5 \mathrm{~mm}$ precargados con una sutura de polipropileno. Con dichos hilos fijamos el borde de la malla en el lado derecho (Fig. 3). A

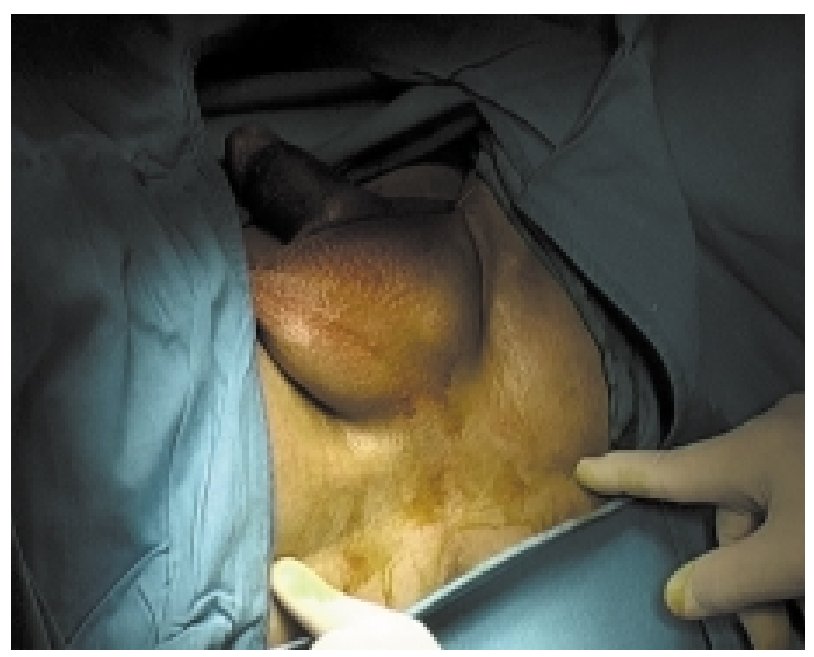

FIGURA 2. Localización de las tuberosidades isquiáticas. 


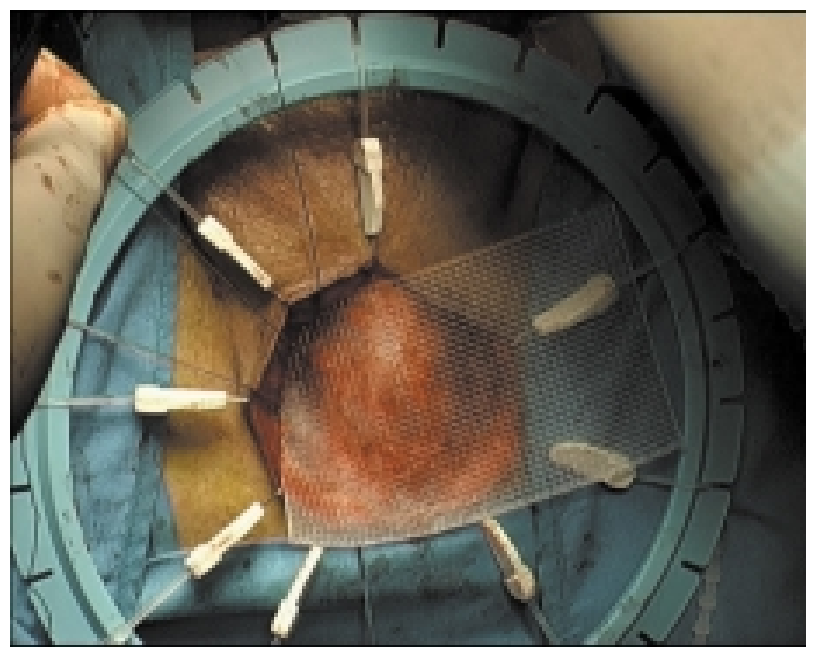

FIGURA 3. Anclaje de la malla en el lado derecho, tras fijar 3 tornillos precargados con hilo de polipropileno.

continuación medimos el espacio entre ambas ramas óseas para determinar el tamaño adecuado de la malla, procediendo a colocar los 3 tornillos que fijaran la malla en el borde izquierdo. Para conocer la tensión de la malla sobre la uretra, colocamos una sonda tipo Foley $18 \mathrm{Ch}$ en fosa navicular, sobre la que se conecta una infusión de suero salino colocada a una altura de 45 $\mathrm{cm}$. por encima de la sínfisis del pubis. Con dicha infusión a ritmo de goteo, se tracciona del borde izquierdo de la malla hasta disminuir o prácticamente abolir dicho goteo, marcando en este momento sobre la malla los puntos donde se fijará a los tornillos (Fig. 4). Posteriormente recortamos la malla sobrante y cerramos mediante sutura perineal por planos, no precisando drenaje (Fig,. 5).

\section{RESULTADOS}

La edad media de los pacientes fue de 65 años, con un tiempo medio libre de enfermedad próximo a 5 años. Los 4 pacientes presentaban un grado de incontinencia moderado (uso de entre 3-5 compresas/día) con importante alteración de su calidad de vida.

Todos los procedimientos se realizaron con raquianestesia, con un tiempo medio de 70 minutos. Las pérdidas hemáticas fueron inapreciables. La estancia postoperatoria fue de 2 días, siendo dados de alta sin sonda vesical y tras comprobar la ausencia de una retención urinaria. No hubo infección de herida quirúrgica ni otras com-

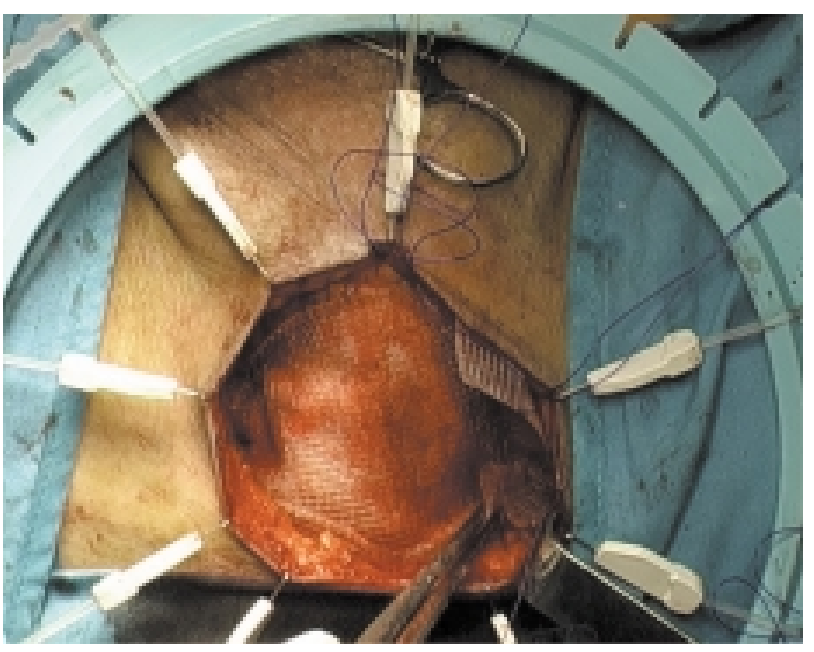

FIGURA 4. Tensión de la malla: tracción del borde izquierdo hasta abolir el goteo de infusión en fosa navicular.

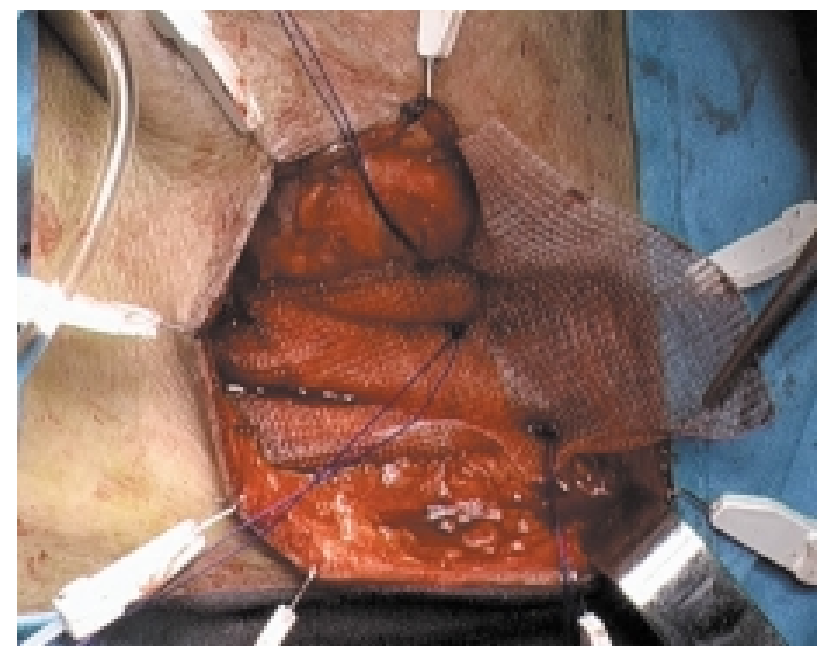

FIGURA 5. Malla en posición bulbouretral (recorte de malla sobrante).

plicaciones postoperatorias. El tiempo de seguimiento medio es de 12,5 meses. En cuanto a continencia dos pacientes presentan continencia total, uno ha mejorado significativamente presentando únicamente algunos escapes con el esfuerzo (uso de una sola compresa al día). En un paciente persiste la incontinencia, ya presente en el postoperatorio inmediato, requiriendo al menos 3 compresas/día. Ningún paciente ha presentado rechazo de la malla. El residuo postmiccional no es significativo (inferior a $30 \mathrm{cc}$ ). Solo un paciente presenta molestias óseas en la zona de anclaje, persistente 11 meses después de la intervención, pero que no obliga a su retirada (Tabla 1). 
Tabla 1

Características de los pacientes. Resultados

\begin{tabular}{ccccccc}
\hline $\begin{array}{c}\text { Paciente } \\
\text { No }\end{array}$ & Edad & $\begin{array}{c}\text { Tiempo desde } \\
\text { prostatectomía } \\
\text { (meses) }\end{array}$ & $\begin{array}{c}\text { Grado de } \\
\text { incontinencia }\end{array}$ & Material & $\begin{array}{c}\text { Tiempo de } \\
\text { seguimiento } \\
\text { (meses) }\end{array}$ & Resultado \\
\hline 1 & 64 & 58 & Moderada & Malla/tornillos & 15 & Continente \\
2 & 66 & 49 & Moderada & INVANCE & 11 & Continente \\
3 & 72 & 63 & Moderada & Malla/tornillos & 17 & Escapes minimos \\
4 & 58 & 55 & Moderada & INVANCE $^{\circledR}$ & 7 & Incontinente \\
\hline
\end{tabular}

\section{DISCUSION}

Una vez superada la intervención radical, los pacientes se enfrentan a aquellos problemas que afectan a su calidad de vida, como son la IUE y la disfunción eréctil. La IUE les afecta de una forma muy especial. La tendencia del urólogo ante este problema es a intentar ser lo menos intervencionista posible. Las medidas conservadoras (ejercicios del suelo pélvico, tratamientos farmacológicos, etc) pueden mejorarla en algunos casos, pero en los mas graves se hace necesario un tratamiento quirúrgico (inyección periuretral endoscópica de sustancias ocupantes de espacio ó bien un EUA). Aunque el EUA constituye actualmente la opción electiva, no está exento de complicaciones y de la necesidad de reintervenciones ${ }^{5}$. Por ello, muchas veces el paciente prefiere convivir con su problema antes de tener que someterse a dicho procedimiento.

En la búsqueda de alternativas, la colocación de una malla suburetral puede parecer al paciente un procedimiento mucho menos complejo, a lo que se suma el tener una mas rápida y fisiológica micción y no requerir el aprendizaje de ningún dispositivo. Existen dos procedimientos de "sling suburetral": el que se realiza con paso de agujas retropúbicas y el que recurre al anclaje sobre las ramas isquiopubianas. Madjar y cols. ${ }^{3}$ sugirieron que la malla anclada a las ramas óseas podía presentar ventajas respecto del anclaje a partes blandas, dada su fijación a una estructura sólida y más resistente.

El sling bulbouretral con paso de aguja retropúbica corregiría la incontinencia al comprimir y elevar la uretral frente a la presión abdominal. Este procedimiento requiere incisión suprapúbica y el paso de agujas lateralmente al cuello vesical y uretra. Este proceso puede producir iatrogenia vesical (perforaciones), si bien un estudio europeo sobre una serie de 36 pacientes refiere solo 2 perforaciones intraoperatorias ${ }^{6}$. En la serie de 64 pacientes de Schaeffer y cols. ${ }^{7}$, el 56\% de pacientes alcanzan una total continencia, mejorando un $8 \%$ de casos (tras una única intervención). En la serie de 6 pacientes de Sousa A y cols $^{8}$, los nuevos cabestrillos de tensión regulable alcanzaban inicialmente un $83 \%$ de curas y un $17 \%$ de mejorías, confirmando posteriormente un $77 \%$ de pacientes secos y un $11 \%$ de mejorías en un estudio multicéntrico presentado en el Congreso Europeo de Estambul $2005^{9}$.

Las primeras experiencias con mallas ancladas son muy recientes. La mayoría de autores obtienen altas tasas de continencia, en torno al 80\% de casos, aunque, con series pequeñas y de cortos seguimientos ${ }^{3,4,9-12}$. En todos los trabajos llama la atención la escasez de complicaciones postoperatorias, no se citan problemas de erosión uretral y el riesgo de osteomielitis parece mínimo. También se ha planteado la incertidumbre sobre los efectos urodinámicos en estos varones, al miccionar frente a una resistencia fija del tracto urinario inferior (TUI). Aunque Clemens y cols ${ }^{13}$ no observaron un patrón miccional obstructivo, para Santos y cols ${ }^{14}$ si existe una resistencia que podría determinar la aparición de complicaciones a largo plazo. En la serie de Ullrich y cols ${ }^{9}$, se analiza especialmente los hallazgos urodinámicos en estos pacientes, tras 25 meses de seguimiento, observando que aunque la resistencia uretral se incrementa de $30,4 \pm 15,9$ a 59,9 \pm 9,7 cm. de agua, no hay cambios significativos en el flujo máximo ni en las presiones del Detrusor, por lo que consideran que dicha resistencia no conduce a obstrucción.

Madjar S y cols. ${ }^{3}$ a principios de esta década publicaron las primeras experiencias en este procedimiento. Sobre una serie de 14 pacientes afectos de IUE post-prostatectomía radical, tras un seguimiento medio de 12,2 meses, 12 casos (86\%) presentaban total continencia y el 14\% restante habían mejorado. No tuvo complicaciones postoperatorias infecciosas ni erosiones uretrales. 
Posteriormente, Comiter $\mathrm{CV}^{4}$, sobre una serie de 21 pacientes y un seguimiento medio de 12 meses, señalaban tasas de curación del 76\% y de mejoría en el 19\%, fracasando únicamente el 5\% de casos. En otra publicación de este mismo grupo, pero a los 25 meses de seguimiento, persistían resultados satisfactorios, al obtener que en el 73\% de casos el problema de la continencia no existía o era mínim ${ }^{9}$. Recientemente Onur y cols. ${ }^{10}$ presentan una serie de 46 varones con IUE sometidos a la colocación de un "sling con anclaje óseo", con una media de seguimiento de 18 meses. Analizan sus resultados en función de las características del paciente, la etiología de la incontinencia, la severidad y el tipo de material utilizado. En su serie el procedimiento falló en 11 pacientes (24\%), el 41\% quedaron secos y el 35\% mejoraron, encontrando como factores desfavorables, la severidad de la incontinencia y el tipo de malla (absorbible). En los pacientes con IUE severa la tasa de curación era de sólo un 50\% de casos, mientras que en la IUE leve-moderada alcanzaba un $83 \%$. Estos resultados parecen permanecer estables con el tiempo, al menos a corto-medio plazo, como pone de manifiesto otra publicación de esta misma serie, tras un seguimiento medio de 24 meses, al observar que el $70 \%$ de pacientes se encontraban satisfechos ${ }^{11}$. El tipo de material también parece influir en los resultados; así Dikranian y cols. ${ }^{12}$ observan que en el grupo de pacientes en los que se coloca una malla de colágeno porcino fracasan el 13\% de casos (el 56\% curaron y 31\% mejoraron), mientras que con material sintético no hubo ningún fracaso (el 87\% curaron y el 13\% mejoraron).

Por tanto, si lo comparamos con el EUA, este procedimiento podría ser muy competitivo, no solo por la mayor sencillez técnica y menor coste, sino por los resultados clínicos. Ullrich y $\operatorname{cols}^{9}$, los autores que mayores estudios han realizado al respecto, consideran que los resultados a medio plazo son perfectamente comparables con el EUA, lo que junto a la baja morbilidad, hace que si se demuestran resultados satisfactorios a largo plazo, este procedimiento podría desbancar al EUA.

\section{CONCLUSIONES}

La colocación de una malla anclada a ramas isquiopubianas constituye un tratamiento prometedor, que puede competir con el EUA en el abordaje de la IUE moderada post-prostatecto- mía radical. Entre sus potenciales ventajas están, la facilidad de ejecución, la buena tolerancia, el mantenimiento de una micción fisiológica, su bajo coste y constituir un procedimiento que el paciente comprende con facilidad. Pero, para determinar su idoneidad, se hacen necesarias series más extensas y evaluar los resultados clínicos a largo plazo.

\section{REFERENCIAS}

1. Gomha MA, Boone TB. Voiding patterns in patients with postprostatectomy incontinence: urodynamic and demographic analysis. J Urol 2003;169:1766-1769.

2. Bosch JL, Klijn AJ, Schroder FH, Hop WC: The artificial urinary sphincter in 86 patients with intrinsic sphincter deficiency: satisfactory actuarial adequate function rates. Eur Urol 2000;38:156160.

3. Madjar S, Jacoby K, Giberti C, Wald M, Halachmi S, Issaq E et al: Bone anchored sling for the treatment of post-prostatectomy incontinence. J Urol 2001;165:72-76.

4. Comiter CV: The male sling for stress urinary incontinence: a prospective study. J Urol 2002;167:597-601.

5. Clemens JQ, Schuster TG, Konnak JW, McGuire EJ, Faerber GJ: Revision rate after artificial urinary sphincter implantation for incontinence after radical prostatectomy: actuarial analysis. J Urol. 2001;166:1372-1375.

6. Sousa A, MRS European Group (Berlin, Genova, Lisboa, Madrid, Milan, Monforte, Thessaloniki). Male Remeex System (MRS) for the treatment of stress urinary incontinence: A multicentric trial. European. J Urol Suppl 4 (2005) no 3 pp 62. Abstract 237.

7. Schaeffer AJ, Clemens JQ, Ferrari M, Stamey TA. The male bulbourethral sling procedure for post-radical prostatectomy incontinence. J Urol. 1998;159:1510-1515.

8. Sousa-Escandon A, Rodriguez Gomez JI, Uribarri Gonzalez C, Marques-Queimadelos A: Externally readjustable sling for treatment of male stress urinary incontinence: points of technique and preliminary results. J Endourol.2004;18:113-118.

9. Ullrich NF, Comiter CV: The male sling for stress urinary incontinence: urodynamic and subjective assessment. J Urol 2004;172: 204-206.

10. Onur R, Rajpurkar A, Singla A. New perineal bone-anchored male sling: lessons learned. Urology. 2004; 64: 58-61.

11. Rajpurkar AD, Onur R, Singla A: Patient satisfaction and clinical efficacy of the new perineal bone-anchored male sling. Eur Urol 2005; 47:237-242.

12. Dikranian AH, Chang JH, Rhee EY, Aboseif SR: The male perineal sling: comparison of sling materials. J Urol 2004;172:608-610.

13. Clemens JQ, Bushman W, Schaeffer AJ: Questionnaire based assessment and urodynamic evaluation following the bulbourethral sling procedure. J Urol 1998;159(Suppl 5):120.

14. Santos J, Payne CK, Ferrari M, Stamey TA: Urodynamics of the modified Stamey procedure. J Urol 1998;159 (Suppl 5):120.

Dr. J.A. Queipo Zaragozá

Calle Santa Teresa 4-8

46530 Puzol (Valencia)

E-mail: queipo@pulso.com

(Trabajo recibido el 13 de abril 2005) 